\title{
MALIGNANT CEREBRAL EDEMA: A RARE PRESENTATION OF NEUROLUPUS
}

Luíza Sá e Rêgo Tupinambá1,*, Mariana Davim Ferreira Gomes', Igor Beltrão Duarte Fernandes, Alexandre Lima Matos1, Edgard Torres dos Reis Neto ${ }^{1}$

1.Universidade Federal de São Paulo, São Paulo (SP), Brazil.

*Corresponding author: luizasrtupinamba@hotmail.com

\section{BACKGROUND}

Neurological manifestations of systemic lupus erythematosus (SLE) can be life threatening conditions. For example, malignant cerebral edema, characterized by diffuse cerebral edema and changes in white matter in the absence of vasculitis is a rare presentation, with only two reported cases, both with fatal outcome. This report describes the third case of this manifestation.

\section{CASE REPORT}

V.C.F.C., 45 years old, was admitted in the emergency department for several holocranial headache four months before, with night awakening and worsening in the decubitus position, without improvement with analgesics. One month before, she presented three episodes of loss of consciousness preceded by diplopia and symptoms of presyncope and followed by long periods of mental confusion and decreased level of consciousness. She was diagnosed with cutaneous articular SLE three years before, without follow-up for one year, using $5 \mathrm{mg}$ prednisone/day only. In the initial assessment, the patient was drowsy with temporal disorientation and impaired evocation memory and ill-defined papilla on fundoscopy. On complementary exams, cerebrospinal fluid was unchanged, except for increased opening pressure (35 mmHg), ESR: 80; anti-DNA $\geq 1 / 320$; complement consumed; positive ribosomal anti-P. A skull computed tomography revealed extensive, diffuse and bilateral hypodensity of the white matter, internal capsule, thalamus, brainstem and cerebellar white matter, associated with diffuse deletion of the intergyral grooves and reduction of the ventricular system. Skull angioresonance revealed hypersignal in the weighted sequences in FLAIR and T2, diffuse, bilateral and symmetrical of the same areas without restriction to diffusion or enhancement to the contrast medium. Black blood protocol without signs of impregnation in the vascular wall. Prednisone $1 \mathrm{mg} / \mathrm{kg}$ was initiated during hospitalization, with improvement in the level of consciousness, but maintenance of headache complaints on Valsalva maneuver occasions. Due to the image pattern compatible with malignant cerebral edema, a rare condition with high lethality, it was decided by pulse therapy with methylprednisolone for three days. The patient was asymptomatic after the first day of pulse therapy. It was decided to perform cyclophosphamide and follow-up in a rheumatology clinic. Three weeks after hospital discharge, the medical team was informed about the patient's clinical worsening, with headache and signs of intracranial hypertension and death in another service.

\section{CONCLUSION}

This is a report of a rare and serious case, deserving scientific communication due to the need to take more aggressive and early approaches in view of the potential lethality of this condition. 